\title{
Article
}

\section{The detectability of Wolf-Rayet Stars in M33-ike spirals up to 30 Mpc}

Pledger, Joanne, Sharp, AJ and Sansom, Anne E

Available at http://clok.uclan.ac.uk/37068/

Pledger, Joanne ORCID: 0000-0001-8907-3051, Sharp, AJ and Sansom, Anne E ORCID: 0000-0002-2782-7388 (2021) The detectability of Wolf-Rayet Stars in M33-ike spirals up to $30 \mathrm{Mpc}$. Monthly Notices of the Royal Astronomical Society, 503 (2). pp. 2168-2178. ISSN 0035-8711

It is advisable to refer to the publisher's version if you intend to cite from the work. http://dx.doi.org/10.1093/mnras/stab630

For more information about UCLan's research in this area go to

http://www.uclan.ac.uk/researchgroups/ and search for <name of research Group>.

For information about Research generally at UCLan please go to http://www.uclan.ac.uk/research/

All outputs in CLoK are protected by Intellectual Property Rights law, including Copyright law. Copyright, IPR and Moral Rights for the works on this site are retained by the individual authors and/or other copyright owners. Terms and conditions for use of this material are defined in the policies page.

\section{CLoK}

Central Lancashire online Knowledge www.clok.uclan.ac.uk

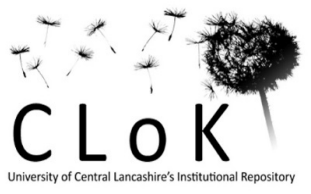




\title{
The detectability of Wolf-Rayet stars in M33-like spirals up to $30 \mathrm{Mpc}$
}

\author{
J. L. Pledger ${ }^{(0)}$, A. J. Sharp and A. E. Sansom ${ }^{(0)}$ \\ Jeremiah Horrocks Institute, University of Central Lancashire, Preston, PR1 2HE, UK
}

Accepted 2021 February 26. Received 2021 February 26; in original form 2020 August 4

\begin{abstract}
We analyse the impact that spatial resolution has on the inferred numbers and types of Wolf-Rayet (WR) and other massive stars in external galaxies. Continuum and line images of the nearby galaxy M33 are increasingly blurred to mimic effects of different distances from 8.4 to $30 \mathrm{Mpc}$, for a constant level of seeing. We use differences in magnitudes between continuum and He II line images, plus visual inspection of images, to identify WR candidates via their ionized helium excess. The result is a surprisingly large decrease in the numbers of WR detections, with only 15 per cent of the known WR stars predicted to be detected at $30 \mathrm{Mpc}$. The mixture of WR subtypes is also shown to vary significantly with increasing distance (poorer resolution), with cooler WN stars more easily detectable than other subtypes. We discuss how spatial clustering of different subtypes and line dilution could cause these differences and the implications for their ages, this will be useful for calibrating numbers of massive stars detected in current surveys. We investigate the ability of ELT/HARMONI to undertake WR surveys and show that by using adaptive optics at visible wavelengths even the faintest $\left(M_{\mathrm{V}}=-3 \mathrm{mag}\right)$ WR stars will be detectable out to $30 \mathrm{Mpc}$.
\end{abstract}

Key words: supernovae: general-stars: Wolf-Rayet-Local Group.

\section{INTRODUCTION}

Evolutionary paths of evolved, massive stars are currently uncertain and this affects our ability to understand which progenitors lead to various categories of core-collapse supernovae (ccSNe; Smith et al. 2011; Eldridge et al. 2013; Groh, Meynet \& Ekström 2013a). This study aims to quantify the effects of spatial resolution on the detection of Wolf-Rayet (WR) stars so that the numbers of massive stars can be correctly interpreted with respect to stellar evolutionary theory.

WR stars are evolved, core-He burning stars with initial masses above $\sim 20 \mathrm{M}_{\odot}$. Most of their hydrogen envelope has been stripped due to violent stellar winds, with mass-loss rates of $\sim 10^{-4}$ $10^{-5} \mathrm{M}_{\odot} \mathrm{yr}^{-1}$ (Nugis \& Lamers 2000). Prominent, broad emission lines arise as a result of these strong winds with speeds of $v$ $\geq 1000 \mathrm{~km} \mathrm{~s}^{-1}$. The metallicity dependence of these stellar winds means that more metal-rich WR stars possess stronger winds and are able to strip even more of their outer envelopes (Vink \& de Koter 2005). WR stars, down to lower masses, can also occur due to mass stripping in close binaries (Götberg, de Mink \& Groh 2017; Shenar et al. 2019), leading to a complexity of evolution. These close binaries may end their lives as merged black holes, high-mass interacting binaries, or as asymmetric supernova explosions, where the black hole gets a kick large enough to prevent a high-mass x-ray binary (HMXB) from forming (Vanbeveren et al. 2020).

As the WR stars undergo stripping they exhibit the products of core-hydrogen burning, namely, helium and nitrogen in their emission-line spectra and are classed as WN stars. More massive, or more metal-rich WR stars undergo enhanced stripping revealing carbon and oxygen produced during core-helium burning and are classed as WC stars (Conti 1976). WR stars can be split into 'early'

^E-mail: jpledger@uclan.ac.uk and 'late' subtypes determined by emission line ratios that represent the temperature of the star. The spectra of cooler, late-type WNL and WCL stars are dominated by stronger NIII and CIII, respectively, compared to NIV and CIV for hotter, early-type WNE and WCE stars, respectively (see Crowther 2007 for a full review of WR classifications).

Current stellar evolutionary models predict that the WC/WN and WR/O ratios increase with metallicity as a result of metal-driven stellar winds during the WR and O star phase (Eldridge \& Vink 2006; Meynet \& Maeder 2005). Theory is currently in disagreement with observational evidence, particularly at higher metallicity where even fewer WN stars are detected than predicted; however, Neugent \& Massey (2011, hereafter NM11) argue that this could simply be a result of WN stars being harder to detect than WC stars. Indeed, when we look at individual WR stars in the Large Magellanic Cloud (LMC; Bibby \& Crowther 2010, their fig. 11), we see that WC stars have the strongest emission lines and when observed through a narrow-band He II filter may appear over 2 mag brighter than when viewed through a continuum filter; we refer to this as emission-line excess. WNE stars have a slightly weaker emission lines excess than WC stars, with the star appearing $\sim 0.5-2$ mag brighter in the He II image compared to a continuum image. WNL stars have the weakest emission line excess of $<1 \mathrm{mag}$ and this creates a natural bias towards detecting WC stars in WR surveys.

However, even in galaxy surveys within the Local Group we are unlikely to be resolving individual WR stars. In large, dense regions the overall brightness is increased but the emission-line excess can be decreased as a result of emission line dilution. For example, in the LMC, based on a spatial scale of $\sim 0.25 \mathrm{pc}$ the R136 cluster has an (unresolved) synthetic narrow-band magnitude of $M_{4686} \sim-$ $10 \mathrm{mag}$ (Bibby \& Crowther 2010) but an excess of only $\mathrm{m}_{4781}-\mathrm{m}_{4686}$ $=0.15 \mathrm{mag}$. Massey \& Hunter (1998) used HST spectroscopy to identify 65 of the bluest, hottest stars, including several H-rich WN 
stars present in R136. Doran et al. (2013) used the VLT-FLAMES Tarantula Survey data to carry out a census of the 30 Doradus starforming region in the LMC, including R136 star cluster, and found that the inner $5 \mathrm{pc}$ hosts $12 \mathrm{WR}$ stars and $\sim 70 \mathrm{O}$ stars. More recently, Crowther et al. (2016) presented new HST data from the R136 central star cluster, classifying the massive stars. Of the 51 stars they obtained HST/STIS spectroscopy of, 24 of them (three WN5, 19 O-type dwarfs and two O-type supergiants) lie within $0.25 \mathrm{pc}$ ( 1 arcsec) of R136a1 (Crowther et al. 2016; their table 4). This spatial resolution is typical of resolutions achieved with ground-based observations, where adaptive optics is not available.

It is the continuum emission from these other blue stars within the same (unresolved) region as the WR stars that causes the strength of the He II $\lambda 4686, \mathrm{~N}$ III $\lambda 4630$, and $\mathrm{C}$ III $\lambda 4650$ emission lines to be diluted. For nearby galaxies such as the LMC (50 kpc) and M33 $(0.84 \mathrm{Mpc})$, where we can resolve sources down to parsec (or subpc) scales, the problem of emission-line dilution is minimal. Beyond the Local Group, this dilution can significantly reduce the emission line excess and severely impact our ability to detect WR stars. A simple, proof-of-concept test by Bibby \& Crowther (2010) estimated that if the LMC stars were located at a distance of $\sim 4 \mathrm{Mpc}$, and the spatial resolution went from 0.25 to $25 \mathrm{pc}$ (assuming a ground-based 1 arcsec seeing), then 20 per cent of the stars would not be detected as a result of line dilution. A full study to quantify the detectability of WR stars, including line dilution, has not been undertaken and this is our aim with the work presented in this paper.

The detectability of WR stars is important not only in the context of stellar evolutionary models but also for identifying the progenitors of Type Ibc ccSNe. Single WN and WC stars have been predicted to be the progenitors of $\mathrm{H}$-poor and $\mathrm{H}+\mathrm{He}$-poor Type $\mathrm{Ib}$ and $\mathrm{Ic}$ ccSNe (Ensman \& Woosley 1988); however; binary star systems can also produce WR stars as a result of Roche Lobe Overflow and common-envelope evolution that would also produce a Type Ib/c ccSNe. (Götberg et al. 2017; Eldridge 2017; Vanbeveren et al. 1998). Moreover, current stellar evolutionary models suggest that the majority of Type Ib/c ccSNe can be produced from close binary stars of much lower initial mass (Dessart et al. 2020).

Over the past two decades attempts have been made to use archival, pre-SN imaging to identify progenitors and confirm a single or binary evolutionary scenario. This has been very successful for hydrogen-rich Type II SN (See Smartt et al. 2009 for a review), but less so for Type Ibc SNe (See Eldridge et al. 2013 and Van Dyk 2017 for a review).

Type Ib SN iPTF13bvn in NGC 5806 at $\sim 22 \mathrm{Mpc}$ is one example. Cao et al. (2013) detected a bright source in archival imaging but concluded that it was too bright for a single WR star. A binary progenitor was favoured by Bersten et al. (2014) based on modelling of the light curve, suggesting that at least some Type Ib SNe result from a binary evolutionary channel. Post-SN HST imaging shows that the progenitor has disappeared (Eldridge \& Maund 2016) and predicts an initial mass of $10-12 \mathrm{M}_{\odot}$ but deep UV observations presented in Folatelli et al. (2016) suggest that the majority of the flux in the pre-SN images came from the SN progenitor itself rather than a binary companion. Most recently, Kilpatrick et al. (2021) combined pre- and post-explosion imaging to identify a progenitor candidate for SN2019yvr that may have experienced significant mass-loss prior to explosion. The cool temperature of the progenitor is inconsistent with the lack of hydrogen in the SN spectra and it remains unclear if the progenitor is a single or binary star.

Progenitors of Type Ic SN remain elusive, with only SN2002ap (Crockett et al. 2007) having a detection limit deep enough to rule out a WR star and support a binary scenario. Similarly, detection limits of post-SN HST imaging of Type Ic SN1994I rule out any binary companion $>10 \mathrm{M}_{\odot}$ (Van Dyk, de Mink \& Zapartas 2016). Such observations were only possible due to the availability of HST imaging and the relatively near distance of the host galaxy, M51 at $\sim 8 \mathrm{Mpc}$. Most recently, Pre-SN HST imaging of SN2017ein in NGC 3938, at an (uncertain) distance of $17-22 \mathrm{Mpc}$, identified a luminous, blue progenitor candidate which could be either a single star, binary system or star cluster (Van Dyk et al. 2018) based on a spatial resolution of $12-16 \mathrm{pc}$.

High-resolution imaging is essential if we are to resolve the single versus binary progenitor debate for ccSNe; this is the motivation for this work. In this paper, we use observations of M33 to investigate the effect that emission-line dilution has on the detectability of WR stars. The most distant supernova progenitor detected is the LBV progenitor of Type IIn SN 2005gl at 66 Mpc (Van Dyk 2017). However, at these distances, it becomes extremely difficult to identify single massive stars and to have a reasonably complete supernova detection rate, thus we set our upper limit at $30 \mathrm{Mpc}$.

In Section 2, we review the WR content of M33. In Section 3, we give the details of our method and analysis followed by the results in Section 4. Section 5 puts our results into context in terms of the predictions from stellar evolutionary models and the implications for detecting supernova progenitors up to $30 \mathrm{Mpc}$.

\section{THE WOLF-RAYET CONTENT OF M33}

M33 (the Triangulum Galaxy) is a face-on, SA(s)cd spiral galaxy at a distance of just $839 \mathrm{kpc}$ (Gieren et al. 2013), and thus is well studied in the literature. Some WR surveys have targeted the giant $\mathrm{H}$ II regions of M33, such as NGC 604 (Bruhweiler, Miskey \& Smith Neubig 2003), NGC 595, and NGC 592 (Drissen et al. 2008); however, the first complete WR survey of the full galaxy was undertaken by NM11 (including earlier work by Massey \& Johnson 1998), and identified 206 WR Stars.

In the NM11 survey, WR candidates were identified using images taken with the Mosaic CCD camera on the Kitt Peak 4.0m Mayall telescope through three narrow-band filters; 'WR He II' $\left(\lambda_{C}=\right.$ $4686 \AA)$, 'WR C III' $\left(\lambda_{C}=4650 \AA\right)$, and a continuum filter 'WR 475' $\left(\lambda_{C}=4750 \AA\right)$. Three fields (each covering $\left.36^{\prime} \times 36^{\prime}\right)$ were obtained per filter to cover the extent of the galaxy's spiral arms and the telescope was dithered between these exposures to fill the chip gaps. The seeing in the central and southern fields was 1.1 arcsec increasing to 1.5 arcsec in the northern field. WR candidates were identified through both photometry and visual inspection of the continuum subtracted He II and C III images. The three-filter approach used in these observations allowed NM11 to not only identify WR candidates but also to predict their WC or WN subtype. This was confirmed via follow-up spectroscopy obtained from the Hectospec instrument on the $6.5 \mathrm{~m}$ Multiple Mirror Telescope.

This catalogue of WR stars was updated by Neugent \& Massey (2014) to add six new WN stars and to declassify an existing object in the 2011 catalogue (an LBV originally identified as a B0.5Ia+WNE system), taking the total known WR population to 211 . The final catalogue consists of $148 \mathrm{WN}$ stars, $52 \mathrm{WC}$ stars, 9 Ofpe/WN9 stars (also known as WN9-11 stars (Crowther, Hillier \& Smith 1995; Crowther \& Bohannan 1997) and two WN/WC transition stars. NM11 consider their survey to be complete to 95 per cent.

\section{METHOD}

The images of M33 from NM11 were kindly provided to us fully reduced by Philip Massey. They covered M33 with three pointings 


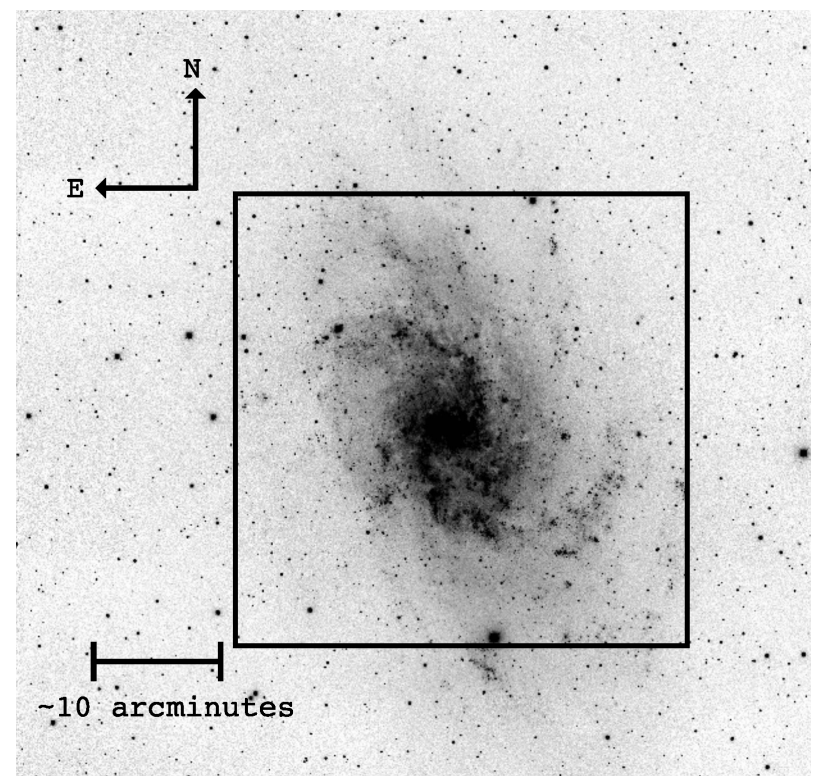

Figure 1. Moses Holden Telescope $B$-band image of the full field of M33 (Credit: M.Norris), showing the field of view covered by the central $36 \times 36$ arcmin pointing of the Kitt Peak He II data from NM11.

and obtained three, $300 \mathrm{~s}$ exposures of each field in the He II $\lambda 4686$, C III $\lambda 4650$, and continuum $\lambda 4750$ filters.

NM11 did not stack these multiple exposures in order to preserve the best photometry possible (Massey et al. 2006). However, to detect the faintest WR stars in more distant galaxies longer exposure times (of order $3000 \mathrm{~s}$ ) are required; therefore, multiple exposures must be stacked. In order to use a consistent approach across all distances, we combined the images from NM11, using the IMCOMBINE routine in IRAF (Tody 1986), as done for WR surveys of more distant galaxies.

For galaxies beyond the Local Group, $8 \mathrm{~m}$ class telescopes are required in order to reach the magnitude limit for WR stars [typically $M_{V}=-3$ mag (Groh et al. 2013b)]. However, C III narrow-band filters are not widely available at these facilities so we chose to only use the $\mathrm{He}$ II and continuum imaging, not the C III images. NM11 used three (central, northern, and southern) pointings to cover the full field of M33 and identify the $211 \mathrm{WR}$ stars but in practice with large facilities one pointing is more achievable. We concentrate our study on the central pointing of M33, the area of which is illustrated in Fig. 1, using a larger scale image taken with the Moses Holden Telescope at UCLan, Preston. This central region contains 196 (93 per cent) of the WR stars.

\subsection{Image degradation}

To investigate the detectability of WR stars out to $30 \mathrm{Mpc}$, we artificially degrade the original M33 image to mimic the observations typical of star-forming spiral galaxies at different distances; this was done using the IRAF routines GAUSS and BLKAVG.

The GAUSS routine convolves the image with a specified Gaussian profile to blur the image. The input width required for the convolution was calculated by using an equation derived from the convolution of two Gaussian functions, which resulted in the formula $\sigma_{g}=$ $\sigma_{M 33} \sqrt{\delta^{2}-1}$, where $\sigma_{M 33}$ is the full width at half-maximum (FWHM) of the stars in the original non-degraded images and $\delta$ $I=D_{\mathrm{deg}} / D_{M 33}$, where $D_{\mathrm{deg}}$ is the distance to which the image was to be degraded to, and $D_{M 33}$ is the distance to M33.
The BLKAVG routine takes $x \times y$ integer pixels in the initial image and bins them to just one pixel in the output image, which takes a value of the mean of the $x \times y$ pixel values in the initial image. In this study, the images were binned by factors of $2 \times 2,5 \times 5$, $10 \times 10,20 \times 20$, and $36 \times 36$, representing galaxy distances of $1.68,4.20,8.39,16.78$, and $30.2 \mathrm{Mpc}$, respectively, for groundbased observations with 1.1 arcsec seeing. The original image can resolve sources to a FWHM $=4.5 \mathrm{pc}$, with the subsequent distances representing spatial resolutions of 9, 22.4, 44.8, 89.6, and $161.1 \mathrm{pc}$, respectively.

Fig. 2 shows the image degradation for two sources in the He II (top), continuum (middle) and continuum subtracted (bottom) images. The central source in the bottom image is a WC4 star and the star to the lower right is a WN6(+abs) star. Both sources start to blend into a single source (along with other stars) at $16.8 \mathrm{Mpc}$.

\subsection{Photometry}

The images of M33, blurred to different spatial resolutions were then analysed to identify the WR population. WR stars exhibit He II emission lines that are stronger than the continuum emission; hence, for a comparison of $\mathrm{He}$ II and continuum filter photometry, we expect WR sources to have $\Delta m<0$ i.e. the star emits more flux in the He II filter than the continuum filter; this is known as the He II excess.

The DAOPHOT package (Stetson, Davis \& Crabtree 1990) within IRAF was used to carry out point spread function (PSF) crowded field photometry on the combined M33 images. Photometry was performed on both the He II $\lambda 4686$ and the Continuum $\lambda 4750$ images, then sources were matched in terms of their $x, y$ co-ordinates and the magnitude difference $\Delta m=m_{4686}-m_{4750}$ calculated for each source. WR sources with $\Delta m<0$ are identified and only accepted as a WR candidate if the He II excess is significant at $\geq 3 \sigma$ level, which we determine using the associated error on the photometry.

\subsection{Inspection of the image}

An additional method for identifying WR candidates in narrow-band imaging is to look for sources with helium excess in the continuum subtracted image by 'blinking' (Massey \& Conti 1983; Moffat \& Shara 1983) which is demonstrated in Fig. 2.

Analysis of the continuum subtracted images is a useful tool for a number of reasons. First, it can help to remove false positives that have no visible helium excess, even though they were determined as having a He II excess from photometry. This is reflected in NM11 and Bibby \& Crowther (2010) who choose a $\Delta m$ cut of 0.1 and $0.15 \mathrm{mag}$, respectively. Secondly, it can show sources of helium excess that the photometry failed to pick up, for example in crowded regions. Finally, the continuum subtracted image can identify WR candidates that are only detected in the He II image and not the continuum; these candidates would not be picked up via photometry.

\section{RESULTS}

We have performed the analysis outlined in Section 3 on the images at $0.84,1.68,4.195,8.39,16.78$, and $30.2 \mathrm{Mpc}$ to quantify the detectability of WR stars at the corresponding spatial resolutions. A summary of our results is presented in Table 1. At the true distance of M33, $0.84 \mathrm{Mpc}$, we detect 93 per cent of the WR stars detected by NM11. Using the a priori information from NM11, the missing WR stars were identified as bad subtractions. Investigating this further, we found that many of the WR stars were identified by our photometry with a small He II excess $(<0.3 \mathrm{mag})$, but they were ruled out as WR 

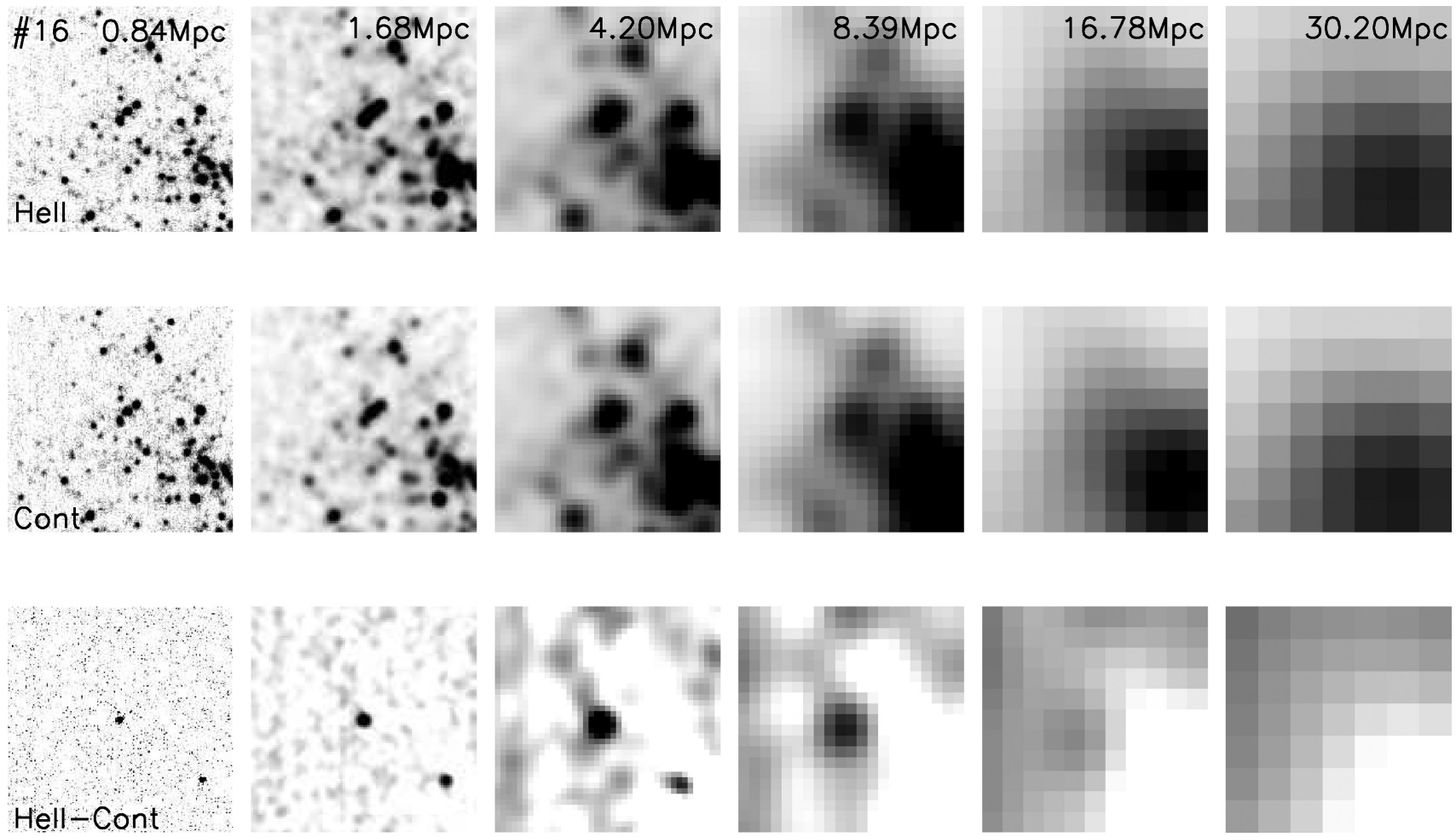

Figure 2. The central source, Source \#16, is a WC4 star detected in the He II filter (top), the Continuum $\lambda 4750$ filter (middle) and the continuum subtracted image (bottom). The source in the lower right of the continuum subtracted image is a WN6 star. The image is degraded to represent the spatial resolution achievable at increasing distances, under the same seeing conditions. The box size corresponds to 1 arcminute diameter at each distance. It is clear that even at $4 \mathrm{Mpc}$ sources merge making it harder to identify the WR stars. The continuum-subtracted image reveals the WC star at $0.84 \mathrm{Mpc}$ without any confusion of surrounding sources and the unresolved star can still be detected in all images within $8.4 \mathrm{Mpc}$. However, at $16.8 \mathrm{Mpc}$ the star cannot be seen in the He II or continuum filters but can be detected in the continuum-subtracted image, but at $30.2 \mathrm{Mpc}$ the WR stars is not detected at all.

Table 1. Results of our analysis at six distances. 93 per cent of the 196 known WR stars in the central pointing are detected in the undegraded (but combined) image decreasing to 15 per cent at $30 \mathrm{Mpc} . N(\mathrm{WR})_{\text {phot }}$ and $N(\mathrm{WR})_{\text {net }}$ are the number of WR stars detected via photometry and additionally from the continuum-subtracted image, respectively. We note that $\mathrm{a} \geq 3 \sigma$ detection was required for the source to be classed as a photometric detection.

\begin{tabular}{lcccc}
\hline $\begin{array}{l}\text { Distance } \\
(\mathrm{Mpc})\end{array}$ & $\begin{array}{c}\text { Spatial } \\
\text { resolution } \\
(\mathrm{pc})\end{array}$ & $N(\mathrm{WR})_{\text {phot }}$ & $N(\mathrm{WR})_{\text {net }}$ & $N(\mathrm{WR})_{\text {total }}$ \\
\hline 0.84 & 4.5 & 172 & 11 & $183(93$ per cent $)$ \\
1.68 & 9.0 & 111 & 47 & $158(81$ per cent $)$ \\
4.20 & 22.4 & 56 & 76 & $132(67$ per cent $)$ \\
8.39 & 44.8 & 2 & 74 & $76(39$ per cent $)$ \\
16.78 & 89.6 & 13 & 35 & $48(24$ per cent $)$ \\
30.20 & 161.1 & 12 & 18 & $30(15$ per cent $)$ \\
\hline
\end{tabular}

candidates after inspection of our continuum subtracted image. These bad subtractions are a result of the PSF being compromised by the combining of our images and is why NM11 chose not to stack their images. At our furthest distance of $30 \mathrm{Mpc}$, or $161 \mathrm{pc}$ resolution, we only detect 15 per cent of the known WR stars in the central pointing of M33, as shown in Fig. 1.

At increasing distances the WR stars can either (i) blend into another source and the emission line is diluted until the WR star is no longer detected (ii) fade into the background noise until it is no longer detectable or (iii) blend with additional WR stars so the emission line excess is increased. In practice, both (i) and (iii) can occur together at some level depending on the distribution of WR stars.

\subsection{Emission line excess}

Fig. 3 shows the magnitude and He II excess emission of the WR stars at each spatial resolution. At high spatial resolutions, we see a range of magnitudes from $M_{4686}=-3.5$ to -9 mag and emission line excess strengths between 0.1 and $3 \mathrm{mag}$. As the spatial resolution decreases, the sources appear to get brighter and the emission line excesses decrease.

At $\sim 8 \mathrm{Mpc}(\sim 45 \mathrm{pc}$ in spatial resolution), all the WR sources have $M_{4686}<-5 \mathrm{mag}$ and $m_{4686}-m_{4750}<0.8 \mathrm{mag}$ (with the exception of one source; Fig. 3d). The emission-line dilution is so severe that many sources end up with $m_{4686}-m_{4750}<0$ and consequently we only identify $\sim 40$ per cent of the WR stars. Many of the WR stars identified are done so through inspection of the continuum subtracted image rather than photometry. This is likely due to increased photometric errors and increased line dilution as a result of crowding that subsequently results in fewer $3 \sigma$ detections.

The farthest distance we investigate is $30 \mathrm{Mpc}$, or an equivalent spatial resolution of $\sim 160 \mathrm{pc}$, where we detect only 15 per cent of WR stars in the central pointing. The magnitude distribution, $M_{4686}$ versus $m_{4686}-m_{4750}$ excess, of the WR stars is shown in Fig. $3 \mathrm{f}$ ). Again, using a priori information, we can see that for the known WR stars that were not detected in our analysis, the majority have $m_{4686}-m_{4750}<0$ mag, suggesting that they are not WR stars. This clearly demonstrates how WR stars can be hidden by surrounding 

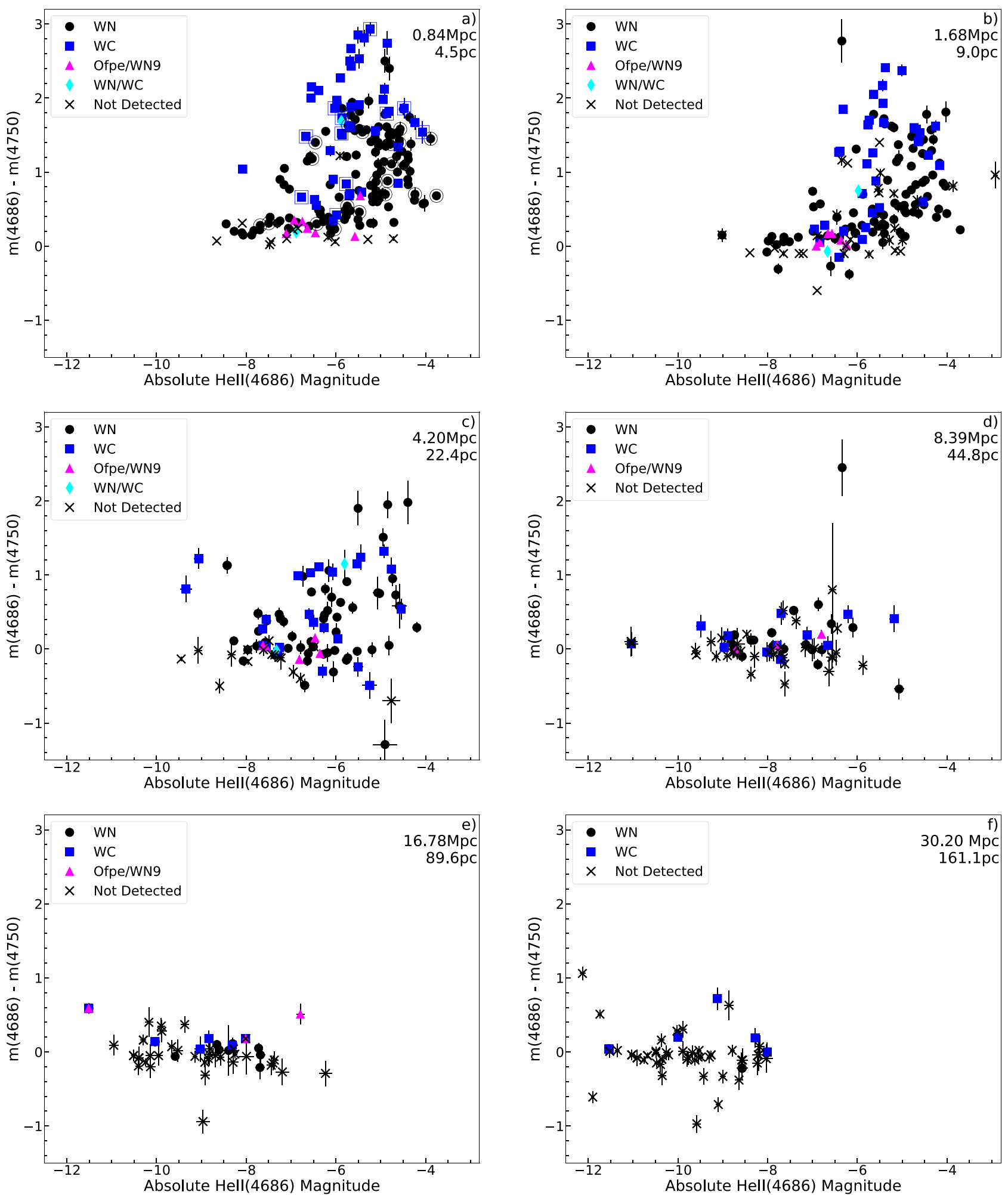

Figure 3. Comparison of $m_{4686}-m_{4750}$ emission line excess versus $\mathrm{M}_{4686}$ for the WR sources in M33 at different spatial resolutions. We note that depending on the WR environment the WR source plotted at each distance may be a single WR star, a binary WR system or an unresolved region containing multiple stars including WR stars; the latter becomes more common as distance increases. Different WR subtypes are highlighted and where a single WR source contains multiple WR stars of different subtypes we have over-plotted both subtypes. We identify binary WC and WN stars from Neugent \& Massey (2014) in a) only by open squares or open circles, respectively. Where possible, photometry (and errors) of the non-detections have been added for completeness using the a priori information from NM11. Many of these sources indicate an excess emission of around 0 mag, demonstrating the effect of line dilution at poorer spatial resolutions. All plots are on the same scale, highlighting the general trend of the WR sources, moving to increased brightness and weaker He II emission line excess as spatial resolution gets worse. These plots exclude WR candidates identified by eye for which no photometry was achieved. 


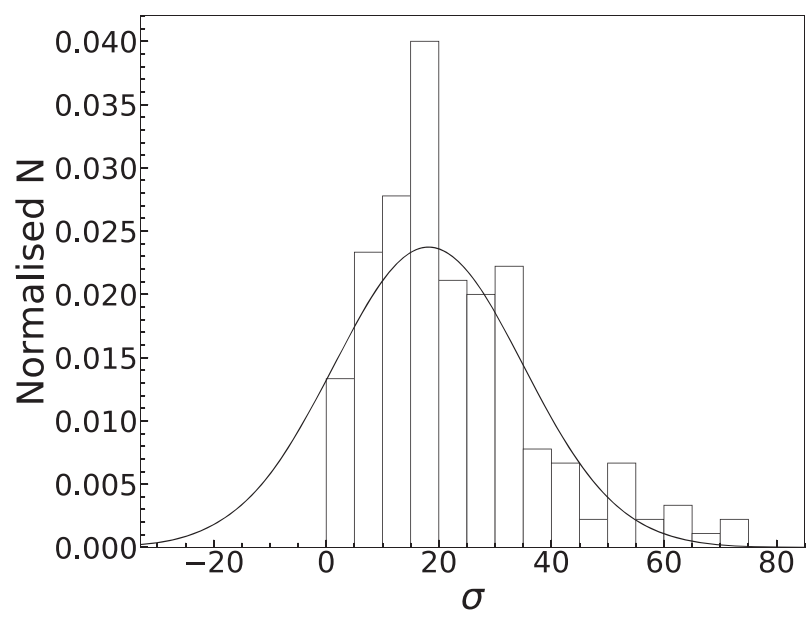

Figure 4. The distribution of the significance of each WR candidate He II excess emission $(\sigma)$ for the original M33 image at $0.84 \mathrm{Mpc}$. A Gaussian profile is fit to show the peak of the distribution at $18 \sigma$.

stars when they cannot be resolved sufficiently. We note that there are three sources with $m_{4686}-m_{4750}>0.5 \mathrm{mag}$, which we would have expected to detect based on their photometry. However, both sources, \#118 with $m_{4686}-m_{4750}=1.06 \pm 0.09$ and source \#57 with $m_{4686}-$ $m_{4750}=0.63 \pm 0.20$ show no He II excess visible in the continuum subtracted image so were discounted as WR candidates at this low resolution, following our candidate criteria. However, source \#54 that has $m_{4686}-m_{4750}=0.72 \pm 0.15$ and a visible excess emission in the continuum subtracted image should have been identified via photometry and has been missed through human error.

We only detect six WR sources at $30 \mathrm{Mpc}$; however, a priori information from NM11 tells us that these six regions host $30 \mathrm{WR}$ stars, suggesting an average of 5 WR stars per source at this lowest resolution. Such information will be useful when estimating the complete WR population of other galaxies. In addition, Fig. (3f) shows that of the six unresolved sources detected at $30 \mathrm{Mpc}$, five host WC stars and only one hosts WN stars but again using a priori information to investigate the contents of each source we see that there are actually more WN stars detected in total than WC stars; this is discussed further in Section 4.4.

\subsection{Significance level of WR candidates}

One criterion for WR candidate selection via photometry was that the $\Delta \mathrm{m}$ He II excess had a significance level greater than $3 \sigma$, i.e. the magnitude of the excess emission was at least three times the error on the excess. This discounted some bonafide WR stars (for example that only had a $2 \sigma$ detection) but if a He II excess was visible then these could be identified as candidates during inspection of the continuum subtracted image. However, if one wanted to survey a galaxy without the manual, time-consuming image inspection, what does our survey tell us about the expected $\sigma$ value for increasing spatial resolutions?

We plotted a histogram of the significance level for every WR candidate identified, whether via photometry or from the continuum subtracted image, and determined the peak of the distribution for each spatial resolution by fitting a Gaussian profile. Fig. 4 shows the distribution for the non-degraded image with a spatial resolution of $\sim 4.5 \mathrm{pc}$. This peak represents the most likely significance of the He II excess of a WR star at that spatial resolution; the results for
Table 2. The peak significance level for a WR source at each spatial resolution. We require a $>3 \sigma$ significance for a source to be classed as a WR candidate. At $30 \mathrm{Mpc}$ there are so few sources detected that the peak of the distribution cannot be determined.

\begin{tabular}{lc}
\hline Resolution $(\mathrm{pc})$ & Peak $\sigma$ \\
\hline 4.5 & 18 \\
9.0 & 15 \\
22.4 & 8 \\
44.8 & 2 \\
89.6 & 1.5 \\
161.1 & - \\
\hline
\end{tabular}

all resolutions are shown in Table 2. It is clear that as the spatial resolution of the image gets poorer the significance of detections reduces. For the original (stacked) image the average WR detection was at $18 \sigma$ falling to $8 \sigma$ at 22.4 pc resolution and below $3 \sigma$ for any resolution above $\sim 40 \mathrm{pc}$. We note that there are so few photometric measurements for WR stars in the $161 \mathrm{pc}(d=30.2 \mathrm{Mpc})$ that no meaningful value of $\sigma$ can be determined.

Our results demonstrate the impact that line dilution can have on detecting WR candidates, decreasing the significance of the He II detection. We find an average $2 \sigma$ detection at $44.8 \mathrm{pc}$ which is consistent with the work of Sandford et al. (2013). They investigate the WR population of NGC 6744 at $11.6 \mathrm{Mpc}$ using VLT FORS imaging of $0.7 \mathrm{arcsec}$, corresponding to a spatial resolution of $\sim 40$ pc. They find that $\sim 40$ per cent of their candidates are detected with a significance level of $3 \sigma, \sim 20$ per cent with a $2 \sigma$ significance. An additional 40 per cent are only detected in the He II image so an excess cannot be determined. Whilst it is possible that their sample is contaminated and not all of their candidates are bonafide WR stars, their and our work both suggest that a $3 \sigma$ significance level for the detections of WR candidates may be too stringent for ground-based WR surveys beyond $\sim 10 \mathrm{Mpc}$. Relaxing the sigma level brings other problems, such as more false positives so a more in depth spectroscopic study of $2 \sigma$ WR candidates is needed to fully understand if WR stars can be detected efficiently at this level.

\subsection{Detection limits of images}

In Table 1 , we note that only 15 per cent of the WR stars in the M33 image degraded to $\sim 160 \mathrm{pc}$ are detected. Fig. 3(f) shows that (most of) the non-detections are due to increased errors and the effect of line dilution decreasing the emission-line excess. However, as we degrade the original image we also increase the background noise causing some of the sources to be indistinguishable from the background. We must ask, are the detection limits of the degraded image sufficient to detect all of the WR population, even if we cannot detect them as individual stars?

By considering all of the sources detected in the M33 images, irrespective of whether they are WR stars, we determine the detection limit of our images. The detection limit of each degraded image is calculated from a histogram of all sources detected in the image, not just WR stars. The 100 per cent completeness limit is noted where the distribution peaks, although as expected some sources are detected at fainter magnitudes (see Bibby \& Crowther (2010) for details). Table 3 reveals that the 100 per cent detection limit of the original image for all stars (including non-WR stars) is $M_{4686}=$ $-2.3 \mathrm{mag}$, increasing in absolute brightness to $M_{4686}=-9.75 \mathrm{mag}$ at $30.2 \mathrm{Mpc}$. Looking at the magnitude distribution of detected WR 
Table 3. The 100 per cent completeness limit as a function of distance. Nearby, we are able to detect fainter objects down to $M_{4686}=-2.3$ mag. At larger distances, these fainter objects are missed due to blending and increased noise.

\begin{tabular}{ll}
\hline Distance $(\mathrm{Mpc})$ & $M_{4686}$ \\
\hline 0.84 & -2.3 \\
1.68 & -2.75 \\
4.20 & -4.5 \\
8.39 & -6.75 \\
16.78 & -8.75 \\
30.20 & -9.75 \\
\hline
\end{tabular}

stars at 30.2 Mpc (Fig. 3f)), 50 per cent of the known WR stars are fainter than this detection limit.

Although we know that at poorer spatial resolution $M_{4686}$ increases in absolute brightness as sources blend, this detection limit at $30.2 \mathrm{Mpc}$ could mean that we are missing WR stars as a result of our images not being deep enough. To quantify these detection statistics, we inspected the $30.2 \mathrm{Mpc}$ image and photometry files and concluded that even though only 30 ( 15 per cent) of the WR stars were identified as WR candidates, 87 per cent of the total WR population was in fact visible in the image, albeit in crowded, unresolved regions with no He II excess. The remaining 13 per cent of WR stars were hidden by the background noise of the image and could potentially be distinguished with increased $\mathrm{S} / \mathrm{N}$. This suggests that at most, with increased exposure times, we could detect and identify $\sim 28$ per cent (15 per cent +13 per cent) of the WR population at a spatial resolution of $160 \mathrm{pc}$.

We note that a detection limit of $M_{4686}=-9.75 \mathrm{mag}$ for the magnitude distribution of WR stars at $0.84,1.69$, and $4.2 \mathrm{Mpc}$ (4$20 \mathrm{pc}$ resolution; Fig. $3 \mathrm{a}-\mathrm{c}$ ) would not be sufficient to detect any of the known WR stars. All the WR sources detected at 30.2 Mpc host multiple WR stars.

\subsection{Detecting different WR subtypes}

So far we have mainly looked at WR detectability of WN and WC stars. In this section, we look in more detail about what this resolution study tells us about the detectability and identification of different $\mathrm{WN}$ and WC subtypes.

Based on the stronger emission lines of WC stars, most WR surveys assume that they are more complete for WC stars than WN stars. To test this we use the known subtype of our detected WR stars (from NM11 and references therein) and we use the classification scheme of Smith (1968) to categorise them as early-WN (WNE) and late-WN (WNL). Table 4 shows the number of each subtype detected at each spatial resolution; those simply noted as 'WN' do not have any detailed classification in NM11. We do not split WC stars into early and late types because there are only two WCL stars in M33. Transition WN/WC and Ofpe/WNL stars are also listed for completeness but low number statistics makes any conclusions unreliable.

Using the a priori information from NM11 for the WR subtype, we can see that in the stacked but undegraded image we detect 98 per cent of the WC stars, 100 per cent of the WNE stars and 90 per cent of the WNL stars. This decreases by $\sim 15$ per cent for each subtype at a spatial resolution of $9 \mathrm{pc}$. At our largest spatial resolution of $161 \mathrm{pc}$ we detect only 14 per cent and 8 per cent of the WC and WNE stars, respectively but are able to recover 40 per cent of the WNL stars.
Table 4. The number of detected sources by subtype at the increasing distances/spatial resolutions. WN stars are those not identified as either WNL or WNE in NM11 and WN/WC are transition stars as defined by Conti \& Massey (1989).

\begin{tabular}{lccccccc}
\hline $\begin{array}{l}\text { Distance } \\
(\mathrm{Mpc})\end{array}$ & $\begin{array}{c}\text { Spatial } \\
\text { Res. (pc) }\end{array}$ & WN & WNE & WNL & WC & $\begin{array}{c}\text { WN/ } \\
\text { WC }\end{array}$ & $\begin{array}{c}\text { Ofpe/ } \\
\text { WNL }\end{array}$ \\
\hline $0.84^{a}$ & 4.5 & 28 & 75 & 31 & 51 & 2 & 9 \\
0.84 & 4.5 & 20 & 75 & 28 & 50 & 2 & 8 \\
1.68 & 9.0 & 18 & 65 & 23 & 43 & 2 & 7 \\
4.20 & 22.4 & 11 & 49 & 23 & 41 & 2 & 6 \\
8.39 & 44.8 & 3 & 29 & 15 & 25 & 1 & 3 \\
16.78 & 89.6 & 2 & 14 & 14 & 14 & 0 & 4 \\
30.20 & 161.1 & 2 & 6 & 12 & 7 & 0 & 3 \\
\hline
\end{tabular}

Note. ${ }^{a}$ In the central pointing of M33 as observed by NM11 (see Section 2 for details).

One explanation is that WNL stars are intrinsically brighter than other WR subtypes (Sander, Hamann \& Todt 2012). However, updated distances from Gaia DR2 has revealed that a correlation between absolute visual magnitude and WR subtype is weak (Hamann et al. 2019; Rate \& Crowther 2020). Moreover, NM11 investigate the average $\mathrm{M}_{V}$ for each individual subtype in the original image and find no distinct differences and a large standard deviation for each subtype. Overall this suggests that the brightness is not responsible for the increased detection of WNL stars at greater distances.

Another possible explanation as to why WNL stars appear to be easier to detect with increasing distance is that they are more concentrated in terms of their spatial distribution across the M33 galaxy compared to other subtypes. This would mean that their emission line excesses, though weak, combine to produce a stronger line that can still be detected at large distances. All of the $12 \mathrm{WNL}$ subtypes detected at an equivalent distance of $30.2 \mathrm{Mpc}$ are located within just two unresolved regions. Fig. 5 shows the H II region NGC 595 in M33 which hosts 9 of the 12 WNL stars detected at $30.2 \mathrm{Mpc}$. At $0.84 \mathrm{Mpc}$ the region is resolved and the He II excess for each star can be quantified, however at $30.2 \mathrm{Mpc}$ the unresolved nature of the region means all WR stars are still detected, albeit in a single region with one He II emission line excess representing all the WR stars. Consequently, despite the emission lines of WNL stars being weaker than WNE and WC stars, their spatial distribution in this region means the $\mathrm{He}$ II excess is still detectable above the continuum making WNL stars more detectable than one would expect at such distances.

Our survey shows that this might indeed be unique to WNL stars. The seven WC stars detected at $30.2 \mathrm{Mpc}$ lie in five unresolved regions, two of which contain no other WR stars. Whilst it is not a surprise that single WC stars can be detected at larger distances given their strong emission line excess, it does suggest WNL stars may have a preference to be in more dense regions/clusters than WC stars.

Smith \& Tombleson (2015) find that Luminous Blue Variables (LBV) in the LMC are more isolated than O stars and WR stars. The explanation for this is that LBVs must result from binary evolution of lower mass stars that have had more time to migrate further from their natal environment. In this work they find also that WR stars are more dispersed than $\mathrm{O}$ stars again having had even a short time to move away from their natal environment, albeit not as far as LBVs. They find that WC stars are more spatially dispersed from $\mathrm{O}$ stars than WN stars, which is consistent with our ability to detect WNL stars in our survey. However Smith \& 

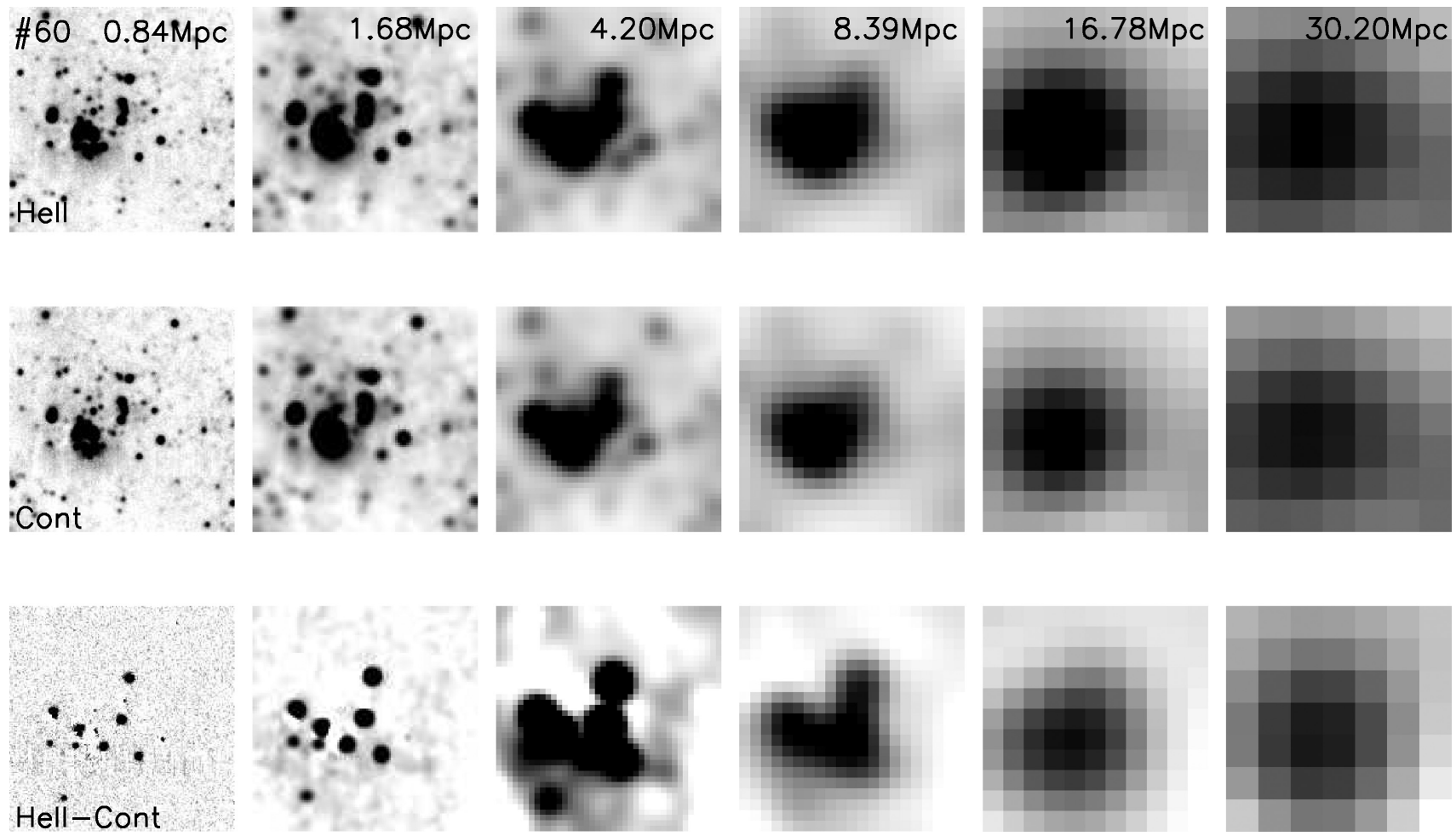

Figure 5. NGC 595 in M33 containing 11 WR stars, including 9 WNL stars, $1 \mathrm{WC}$ and $1 \mathrm{WN}$ star. This figure shows the He II (top), continuum (middle) and continuum-subtracted (bottom) image for a $\sim 1$ arcminute box around the region. The image is degraded from $0.84 \mathrm{Mpc}$ to $30.2 \mathrm{Mpc}$ (left to right), showing how the individual WR sources become part of the a single source at higher distances. The He II excess (shown as dark emission in the bottom row) can be detected out to $30.2 \mathrm{Mpc}$.

Tombleson (2015) interpret this as WC stars being older than WN stars, rather than more massive (younger) and they do not investigate whether the different distributions of WN stars relative to $\mathrm{WC}$ stars is statistically significant. Further investigation into the spatial distribution of WR subtypes is required to see if any clumping of individual subtypes is truly present and subsequently if this leads to a higher detection rate for a specific subtypes e.g. WNL stars as this work suggests.

\section{DISCUSSION}

\subsection{Comparisons with stellar evolutionary models}

WR stars are produced by the removal of the outer envelope of a star by metal-driven stellar winds. Consequently, more WR stars are found in metal-rich regions because the stellar winds are more efficient at stripping the stars. This causes the lower mass limit for a WR star to decrease, with some stars that would normally become Red Supergiants now becoming WN stars. Similarly, the mass limit for a WC star decreases so the WC/WN ratio changes with metallicity (Eldridge \& Vink 2006). However, direct comparison of observational results with stellar evolutionary models has revealed a higher observed WC/WN ratio than predicted across all metallicities. Most studies (e.g. Crowther et al. 2003; NM11; Bibby \& Crowther 2012) correct for completeness in terms of an absolute magnitude limit and an observational bias towards WC stars to account for the discrepancy. Fig. 6 adapted from Bibby \& Crowther (2010) shows that correcting for completeness in this way reduces the observed ratio towards the predicted values (solid points to open

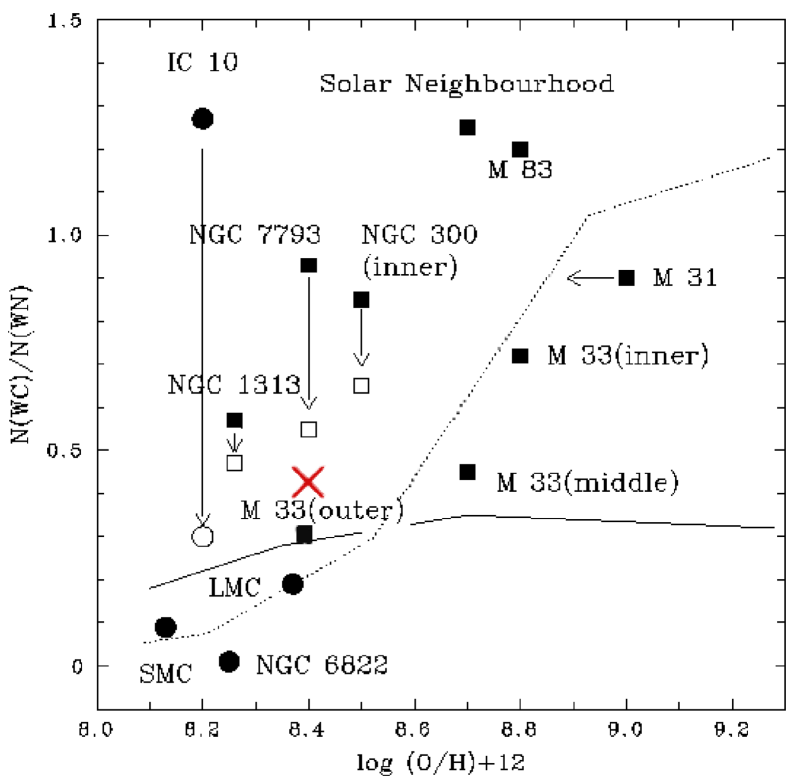

Figure 6. Adapted from Bibby \& Crowther (2010; their fig. 13a) showing the $\mathrm{WC} / \mathrm{WN}$ ratio in a number of galaxies at different metallicities. The observed ratios are plotted as solid points and the ratio corrected for WC bias and detection limits are the open points. The WC/WN ratio for NGC 7793 is additionally corrected for spatial resolution and line dilution using this work and is indicated by the red X. The result for NGC 7793 is more in line with evolutionary predictions from Eldridge \& Vink (2006)(dotted line) and Meynet \& Maeder (2005) (solid line). 


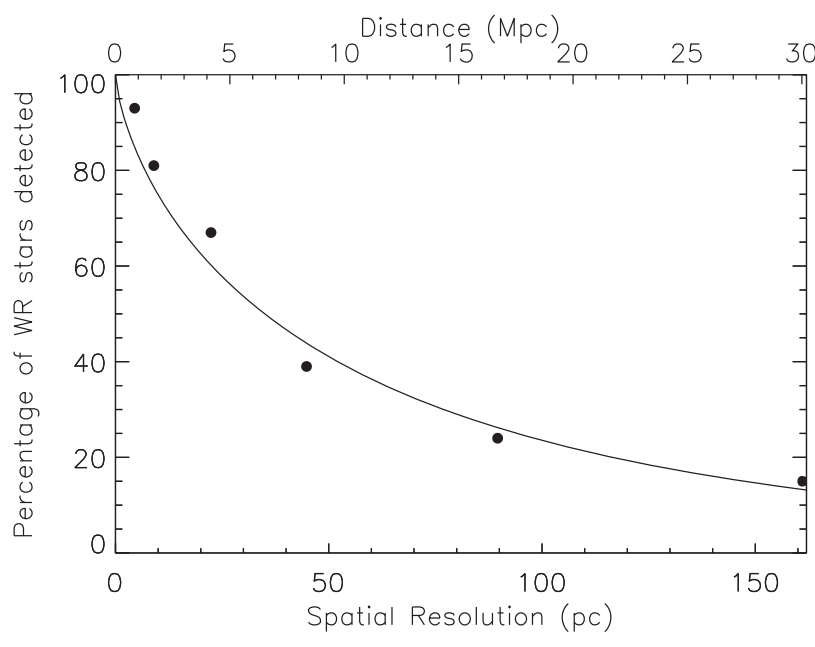

Figure 7. Results of the image degradation showing the percentage of WR stars detected at increasing distance and decreasing spatial resolution as listed in Table 1.

points) but theoretical predictions are still not in agreement with observations.

Most recently, Stanway, Eldridge \& Chrimes (2020) use the WC/WN ratio to investigate the binary fraction of the stellar population and whether this can be recovered from observations of stellar populations. They find that the WC/WN ratio decreases sharply with increasing binary fraction but note that number ratios from unresolved stellar populations must be used with caution when comparing to stellar models.

If the detectability of WN and WC stars is not equally affected by line dilution, then this will have an impact on measured WC/WN ratios and any conclusions from comparison with stellar evolutionary models. The work presented here may be able to provide a further correction accounting for spatial resolution. For example, if we consider the WR analysis of NGC 7793 for which the relevant data is available, Bibby \& Crowther (2010) observe a WC/WN ratio of $\sim 0.95$, decreasing to $\sim 0.5$ after applying standard completeness corrections. This is still not in agreement with stellar evolutionary models from which we expect the a ratio of $\sim 0.2-0.3$. The resolution of the VLT/FORS1 NGC 7793 data is 1.3 arcsec, corresponding to a physical scale of $\sim 25 \mathrm{pc}$ assuming a distance of $3.91 \mathrm{Mpc}$. From our analysis of M33 (see Table 4) if we exclude transition $\mathrm{WN} / \mathrm{WC}$ and Ofpe/WNL stars, we see that the WC/WN ratio at a resolution of $22.4 \mathrm{pc}$ (distance of $4.2 \mathrm{Mpc}$ ) is 0.49 , whereas at the best spatial resolution of $4.5 \mathrm{pc}$ we find $\mathrm{WC} / \mathrm{WN}=0.38$. If this 0.11 correction is applied to the NGC 7793 data then this suggests (see red X on Fig. 6) that about half of the discrepancy between theoretical predictions and observations can be accounted for by WR stars not detected due to line dilution (rather than due to magnitude limits).

\subsection{Implications for supernova progenitor detections}

Fig. 7 shows the percentage of WR stars that you would expect to detect as a function of spatial resolution and distance, resulting from our analysis. The WR stars in this figure are those detected at that spatial resolution via photometry or inspection of the image, as outlined in Section 3. Each WR that is detected with a He II excess is included, even if there are multiple WR stars within one unresolved source.
These data are empirically fit by equation (1) which constrains the fit to 100 per cent at a spatial resolution of $0 \mathrm{pc}$, and is applied up to a spatial resolution of $160 \mathrm{pc}$. This equation, plus the scatter about it seen in Fig. 7, can be used to give an estimate of completeness as a function of spatial resolution which is useful when planning observations.

$y=100 \times 10^{-0.025 x^{0.7}}$.

It is clear that beyond a spatial resolution of $\sim 20 \mathrm{pc}$, typical of a classical H II region (Conti, Crowther \& Leitherer 2008), we cannot detect a sufficient ( $>60$ per cent) number of WR stars to consider a survey complete. At ground-based resolution of $\sim 1$ arcsec, $20 \mathrm{pc}$ corresponds to $\sim 4 \mathrm{Mpc}$, which limits the number of galaxies we can survey to a reasonable degree of completeness. This distance limit drastically reduces the probability of detecting a preSN WR star; there have been no Type Ibc ccSN within $\sim 4 \mathrm{Mpc}$ within the last $10 \mathrm{yr}$, and only 4 since on record in total [SN1954A, SN1962L, SN1983N, SN2002ap, and SN2008dv (Guillochon et al. 2017)].

However, if $H S T$ or Adaptive Optics is utilized for WR surveys then the 0.1 arcsec resolution affords a factor of $\sim 10$ increase in distance, out to $40 \mathrm{Mpc}$. Within this distance, 138 Type Ibc SNe have been recorded (Barbon et al. 1999; Guillochon et al. 2017) that is a significant increase on $4 \mathrm{Mpc}$ and would increase our chances of identifying a pre-SN WR star if such WR surveys existed.

From Table 1, it is clear that visual inspection of the image results in a more complete WR survey. Whilst Morello et al. (2018) successfully use a machine-learning approach to identify new WR stars in the Milky Way, it is unclear if such an approach can be successfully employed for more distant galaxies where we see changing photometric properties with changing spatial resolution (recall Fig. 3).

\subsection{Implications for current and future surveys}

Here, we consider how detectable WR stars are at large distances based on both spatial resolution and magnitude, accounting for current and upcoming instrumentation. We set a threshold of $M_{V}$ $=-3$ mag for the faintest WR stars which is consistent with the analysis of WR stars in the LMC, SMC, and M33 by Neugent \& Massey (2011) and determine $m_{V}$ for seven distances up to $60 \mathrm{Mpc}$. Based on our previous experience, we assume an exposure time of $1 \mathrm{hr}$ and use the narrow-band VLT/FORS2 side-band filter, He II/6500 + 49 $9^{1}$ centred on $4781 \AA$ with the standard resolution collimator to calculate the $\mathrm{S} / \mathrm{N}$ ratio achievable in the continuum for the faintest WR stars. We also look at VLT/MUSE IFU in Wide Field Mode (WFM) with extended spectral range to include He II $\lambda 4686$ line. The Muse User Manual ${ }^{2}$ gives the relevant parameters for no AO WFM with 0.2 arcsec per pixel sampling. Overall the read out and dark count contribution to the noise is small and the noise is sky dominated under grey observing conditions. Sky values for the VLT are taken from Noll et al. (2012) and are assumed to be similar at the ELT site.

We assume a nominal resolution of 0.8 arcsec for VLT and 0.06 arcsec for ELT based on a 20 mas sampling as suggested

\footnotetext{
${ }^{1}$ http://www.eso.org/sci/facilities/paranal/instruments/fors/doc/VLT-MANESO-13100-1543_P06.pdf

${ }^{2}$ http://www.eso.org/sci/facilities/paranal/instruments/muse/doc/ESO-2616 50_MUSE_User_Manual.pdf
} 
Table 5. Single WR continuum detectability with spatial resolutions and signal-to-noise values achievable for observations of WR stars up to $\sim 60 \mathrm{Mpc}$. We present data for observations for VLT/FORS2 without AO, VLT/MUSE without AO in extended mode and ELT/HARMONI with AO. FORS2 and MUSE have a resolution of 0.8 arcsec, whilst HARMONI has a resolution of 0.06 arcsec. We assume $\mathrm{M}_{V}=-3$ mag for the faintest WR stars and grey sky background.

\begin{tabular}{|c|c|c|c|c|c|c|c|c|c|}
\hline $\begin{array}{l}\text { Scale } \\
\left(\mathrm{pc} \operatorname{arcsec}^{-1}\right)\end{array}$ & $\begin{array}{l}\text { Distance } \\
(\mathrm{Mpc})\end{array}$ & $\begin{array}{c}\text { Example } \\
\text { Environment }\end{array}$ & $\begin{array}{l}m-M \\
(\mathrm{mag})\end{array}$ & $\begin{array}{l}\mathrm{m}(\mathrm{V}) \\
(\mathrm{mag})\end{array}$ & $\begin{array}{c}\mathrm{S} / \mathrm{N} \\
\text { FORS2 } \\
\text { (no } \mathrm{AO} \text { ) }\end{array}$ & $\begin{array}{c}\text { S/N } \\
\text { MUSE } \\
(\text { no AO - E) }\end{array}$ & $\begin{array}{l}0.8 \text { arcsec Resolution } \\
(\mathrm{pc} \text { resolution }\end{array}$ & $\begin{array}{c}\text { S/N } \\
\text { HARMONI } \\
\text { (with AO) }\end{array}$ & $\begin{array}{l}0.06 \text { arcsec Resolution } \\
\quad\left(\mathrm{pc} \text { resolution }{ }^{-1}\right)\end{array}$ \\
\hline 8.14 & 1.68 & & 26.13 & 23.13 & 45.80 & 24.37 & 6.52 & 652.81 & 0.49 \\
\hline 20.36 & 4.20 & Sculptor Group & 28.12 & 25.12 & 7.51 & 3.99 & 16.29 & 213.38 & 1.22 \\
\hline 40.68 & 8.39 & & 29.62 & 26.62 & 1.89 & 1.00 & 32.54 & 72.12 & 2.44 \\
\hline 81.35 & 16.78 & Fornax Cluster & 31.12 & 28.12 & 0.47 & 0.25 & 65.08 & 20.26 & 4.88 \\
\hline
\end{tabular}

by specification document for ELT-IFU observations in the visible range. ${ }^{3}$ The throughput of the IFU in the visible range is the least certain aspect of our analysis which we take as $\sim 30$ per cent ${ }^{4}$ compared to $\sim 60$ per cent for VLT/FORS2 with the He II filter and $\sim 17$ per cent for VLT/MUSE.

The results of our analysis are presented in Table 5. Using VLT with FORS2 or MUSE without adaptive optics, we expect to be able to detect the faintest $\left(M_{V}=-3\right)$ WR stars out to a distance of $\sim 4.5 \mathrm{Mpc}$ in terms of $\mathrm{S} / \mathrm{N}$. However, at this distance the spatial resolution of $\sim 16 \mathrm{pc}$ would likely hinder our ability to detect and resolve all WR stars as indicated by Fig. 7. This is consistent with previous WR surveys at similar distances (Hadfield et al. 2005; Bibby $\&$ Crowther 2010, 2012). Some WR and WR star complexes have been detected with MUSE [e.g. in NGC 300 at $1.9 \mathrm{Mpc}$ (Roth et al. 2018); in NGC 4038/39 at 18 Mpc (Gómez-González et al. 2021)]. Looking to future instrumentation, namely ELT/HARMONI with adaptive optics available at visible wavelengths, we expect to be able to achieve sufficient $\mathrm{S} / \mathrm{N}$ to detect many individual WR stars out to $30 \mathrm{Mpc}$. Such future observations would allow for studies of WR populations in many different types of galaxies, in different environments.

\section{CONCLUSIONS}

We present a WR survey of M33 using narrow-band images degraded to five different absolute spatial resolutions, representative of distances of up to $30 \mathrm{Mpc}$ for 1.1 arcsec apparent resolution. We find the following:

1) Emission line dilution resulting from poorer spatial resolution drastically reduces the detectability of WR stars out to $30 \mathrm{Mpc}$.

2) Based on the distribution of the significance level of the He II excess for each WR candidate detection, we conclude that inspection of the image by eye is vital if we are to detect as many WR stars as possible.

3) Resolution plays more of a role in limiting the detection of WR stars than $\mathrm{S} / \mathrm{N}$.

4) WNL stars appear to be more clustered compared to WC stars, suggesting that WC stars are older than WNL stars, rather than more massive.

5) If a correction for line dilution as a result of spatial resolution is applied, WC/WN ratios are more in line with predictions from stellar evolutionary models.

\footnotetext{
${ }^{3}$ https://www.eso.org/sci/facilities/eelt/docs/ESO-191883_2_Top_Level_Req uirements_for_ELT-IFU.pdf

${ }^{4}$ www2.physics.ox.ac.uk/research/visible-and-infrared-instruments/harmoni
}

In summary, the emission-line dilution that occurs as a result of being unable to resolve bonafide WR stars from their neighbours severely impacts our ability to identify these stars. This has a wider impact on identifying supernova progenitors as well as testing predictions from stellar evolutionary models.

Narrow-band imaging and spectroscopic surveys of galaxies expected to have a significant WR population (e.g. NGC 6946, M83, and M51) have already been undertaken with ground-based facilities. These surveys are typically limited to $\sim 10 \mathrm{Mpc}$ for the reasons quantified in this paper. $\mathrm{M} 101$, at $6.5 \mathrm{Mpc}$, is the only complete grand spiral galaxy to be imaged with HST/WFPC3 using F469N narrow-band filters to isolate the He II emission. HST affords a similar spatial resolution to the undegraded M33 imaging of $\sim 3 \mathrm{pc}$ and reveals more WR candidates than would be expected from similar ground-based surveys (Shara et al. 2013).

We show that current instrumentation, e.g. VLT/FORS2 or MUSE limits WR surveys to within $4 \mathrm{Mpc}$ and that currently only narrowband imaging with $H S T / \mathrm{WFC} 3$, can produce the superior spatial resolution required to detect a significant number of WR stars out to larger distances. We investigate the ability of planned instruments such as ELT/HARMONI, with AO available at optical wavelengths and conclude that such instruments will significantly improve our ability to detect WR stars and allow us to undertake WR surveys of galaxies at distances of up to $30 \mathrm{Mpc}$.

\section{ACKNOWLEDGEMENTS}

The authors thank Philip Massey and Kathryn Neugent for providing them with the fully reduced narrow-band imaging of M33. We thank the referee, Paul Crowther, for providing helpful comments that improved this paper. We acknowledge Aaron Brocklebank for the initial degrading of the images with financial support from STFC. AJS acknowledges financial support from the UCLan Undergraduate Research Internship Programme 2019. We also thank Molly Hawkin from Cardinal Newman College for her work on Figs 2 and 5 as part of the Ogden Trust outreach programme. This paper makes use of observations obtained with the University of Central Lancashire's Moses Holden Telescope.

\section{DATA AVAILABILITY}

The data underlying this article will be shared on reasonable request to the corresponding author. 


\section{REFERENCES}

Barbon R., Buondi V., Cappellaro E., Turatto M., 1999, A\&A, 139, 531

Bersten M. C. et al., 2014, AJ, 148, 68

Bibby J. L., Crowther P. A., 2010, MNRAS, 405, 2737

Bibby J. L., Crowther P. A., 2012, MNRAS, 420, 3091

Bruhweiler F. C., Miskey C. L., Smith Neubig M., 2003, AJ, 125, 3082

Cao Y. et al., 2013, ApJ, 775, L7

Conti P., 1976, in Proc. 20th Colloq. Int. Ap. University of Liege, Liege, p. 193

Conti P. S., Massey P., 1989, ApJ, 337, 251

Conti P. S., Crowther P. A., Leitherer C., 2008, From Luminous Hot Stars to Starburst Galaxies. Cambridge Univ. Press, Cambridge, UK

Crockett R. M. et al., 2007, MNRAS, 381, 835

Crowther P. A. et al., 2016, MNRAS, 458, 624

Crowther P. A., 2007, ARA\&A, 45, 177

Crowther P. A., Bohannan B., 1997, A\&A, 317, 532

Crowther P. A., Hillier D. J., Smith L. J., 1995, A\&A, 293, 172

Crowther P. A., Drissen L., Abbott J. B., Royer P., Smartt S. J., 2003, A\&A, 404, 483

Dessart L., Yoon S.-C., Aguilera-Dena D. R., Langer N., 2020, A\&A, 642, A106

Doran E. I. et al., 2013, A\&A, 558, A134

Drissen L., Crowther P. A., Úbeda L., Martin P., 2008, MNRAS, 389, 1033

Eldridge J. J., 2017, Population Synthesis of Massive Close Binary Evolution. Springer International Publishing, p. 671

Eldridge J. J., Maund J. R., 2016, MNRAS, 461, L117

Eldridge J. J., Vink J. S., 2006, A\&A, 452, 295

Eldridge J. J., Fraser M., Smartt S. J., Maund J. R., Crockett R. M., 2013, MNRAS, 436, 774

Ensman L. M., Woosley S. E., 1988, ApJ, 333, 754

Folatelli G. et al., 2016, ApJ, 825, L22

Gieren W. et al., 2013, ApJ, 773, 69

Gómez-González V. M. A., Mayya Y. D., Toalá J. A., Arthur S. J., ZaragozaCardiel J., Guerrero M. A., 2021, MNRAS, 500, 2076

Götberg Y., de Mink S. E., Groh J. H., 2017, A\&A, 608, A11

Groh J. H., Meynet G., Ekström S., 2013a, A\&A, 550, L7

Groh J. H., Meynet G., Georgy C., Ekström S., 2013b, A\&A, 558, A131

Guillochon J., Parrent J., Kelley L. Z., Margutti R., 2017, ApJ, 835, 64

Hadfield L. J., Crowther P. A., Schild H., Schmutz W., 2005, A\&A, 439, 265

Hamann W. R. et al., 2019, A\&A, 625, A57

Kilpatrick C. D. et al., 2021, MNRAS, preprint (arXiv:2101.03206)

Massey P., Conti P. S., 1983, ApJ, 273, 576

Massey P., Hunter D. A., 1998, ApJ, 493, 180

Massey P., Johnson O., 1998, ApJ, 505, 793
Massey P., Olsen K. A. G., Hodge P. W., Strong S. B., Jacoby G. H., Schlingman W., Smith R. C., 2006, AJ, 131, 2478

Meynet G., Maeder A., 2005, A\&A, 429, 581

Moffat A. F. J., Shara M. M., 1983, ApJ, 273, 544

Morello G., Morris P. W., Van Dyk S. D., Marston A. P., Mauerhan J. C., 2018, MNRAS, 473, 2565

Neugent K. F., Massey P., 2011, ApJ, 733, 123 (NM11)

Neugent K. F., Massey P., 2014, ApJ, 789, 10

Noll S., Kausch W., Barden M., Jones A. M., Szyszka C., Kimeswenger S., Vinther J., 2012, A\&A, 543, A92

Nugis T., Lamers H. J. G. L. M., 2000, A\&A, 360, 227

Rate G., Crowther P. A., 2020, MNRAS, 493, 1512

Roth M. M. et al., 2018, A\&A, 618, A3

Sander A., Hamann W. R., Todt H., 2012, A\&A, 540, A144

Sandford E., Bibby J. L., Zurek D., Crowther P., 2013, Bulletin of the American Astronomical Society, American Astronomical Society Meeting Abstracts \#221. p. 443.06

Shara M. M., Bibby J. L., Zurek D., Crowther P. A., Moffat A. F. J., Drissen L., 2013, AJ, 146, 162

Shenar T. et al., 2019, A\&A, 627, A151

Smartt S. J., Eldridge J. J., Crockett R. M., Maund J. R., 2009, MNRAS, 395, 1409

Smith L. F., 1968, MNRAS, 138, 109

Smith N., Tombleson R., 2015, MNRAS, 447, 598

Smith N., Li W., Filippenko A. V., Chornock R., 2011, MNRAS, 412, 1522

Stanway E. R., Eldridge J. J., Chrimes A. A., 2020, MNRAS, 497, 2201

Stetson P. B., Davis L. E., Crabtree D. R., 1990, in Jacoby G. H., ed., ASP Conf. Ser. Vol. 8, CCDs in Astronomy. Astron. Soc. Pac., San Francisco, p. 289

Tody D., 1986, in Crawford D. L., ed., SPIE Conf. Ser. Vol. 627. SPIE, Bellingham, p. 733

Van Dyk S. D. et al., 2018, ApJ, 860, 90

Van Dyk S. D., 2017, Phil. Trans. R. Soc. London Ser. A, 375, 20160277

Van Dyk S. D., de Mink S. E., Zapartas E., 2016, ApJ, 818, 75

Vanbeveren D., De Donder E., Van Bever J., Van Rensbergen W., De Loore C., 1998, New Astron., 3, 443

Vanbeveren D., Mennekens N., van den Heuvel E. P. J., Van Bever J., 2020, A\&A, 636, A99

Vink J. S., de Koter A., 2005, A\&A, 442, 587

This paper has been typeset from a $\mathrm{T}_{\mathrm{E}} \mathrm{X} / \mathrm{LT} \mathrm{E} \mathrm{X}$ file prepared by the author. 